\title{
Consecuencias de las variaciones microclimáticas sobre la visita de insectos polinizadores en dos especies de Chaetanthera (Asteraceae) en los Andes de Chile central
}

\author{
Consequences of microclimate variation on insect pollinator visitation in two species of \\ Chaetanthera (Asteraceae) in the central Chilean Andes
}

\author{
CRISTIAN TORRES-DÍAZ ${ }^{1,3}{ }^{*}$, LOHENGRIN A. CAVIERES $^{1,3}$, CARLOS MUÑOZ-RAMÍREZ $^{1}$ \\ \& MARY T. K. ARROYO 2,3
}

\footnotetext{
${ }^{1}$ Departamento de Botánica, Facultad de Ciencias Naturales y Oceanográficas, Universidad de Concepción, Casilla 160-C, Concepción, Chile

${ }^{2}$ Departamento de Ciencias Ecológicas, Facultad de Ciencias, Universidad de Chile ${ }^{3}$ Instituto Milenio de Ecología y Biodiversidad (IEB), Las Palmeras 3425, Santiago, Chile;

* e-mail para correspondencia: critorre@udec.cl
}

\begin{abstract}
RESUMEN
La actividad de polinizadores puede ser afectada por factores bióticos (e.g., densidad floral en el parche, despliegue floral) o por factores abióticos (e.g., temperatura, velocidad de viento, nubosidad). A pesar de la notable variación local, estacional e interanual en temperatura en zonas alpinas, las consecuencias de la variación microclimática local sobre la actividad de los polinizadores han recibido escasa atención en ambientes de alta montaña. En este estudio comparamos las tasas de visita a flores de polinizadores realizadas sobre poblaciones de exposición norte y oeste, en Chaetanthera apiculata $(3.100 \mathrm{~m}$ de altitud), y en poblaciones de exposición este y oeste en C. lycopodioides $(3.300 \mathrm{~m}$ de altitud). Se estudiaron los sistemas de reproducción de ambas especies para determinar su nivel de dependencia de un agente de polinización externo. En C. apiculata, la población que crece sobre la ladera de exposición norte estuvo sujeta a mayores temperaturas diurnas $\left(1,8^{\circ} \mathrm{C}\right)$ y mayores tasas de visita $(7,8$ veces $)$ que la población sobre la ladera de exposición oeste. En C. lycopodioides, a su vez, la ladera de exposición este fue más cálida $\left(3{ }^{\circ} \mathrm{C}\right)$ y más visitada (cuatro veces) que la población de exposición oeste. Mientras en $C$. apiculata, solo Faunula leucoglene (Lepidóptera: Satyridae) presentó mayor actividad en la población más cálida, en $C$. lycopodioides, solo F. leucoglene y Liphantus sp. (Himenóptera: Andrenidae) tuvieron mayor actividad en la población más cálida. La tasa de visitas de Faunula leucoglene fue afectada por la temperatura en $C$. apiculata y $C$. lycopodioides. Ambas especies, C. apiculata y C. lycopodioides, son parcialmente autocompatibles, y por lo tanto requieren polen exógeno para alcanzar una producción de semillas máxima. Nuestros resultados muestran que en ambientes de alta montaña las tasas de visita de polinizadores son altamente variables y que los factores microclimáticos pueden generar más variación en las tasas de visitas a flores que la densidad de capítulos florales en los parches. Nuestros resultados aportan valiosa información sobre las potenciales consecuencias del cambio climático global en la polinización.
\end{abstract}

Palabras clave: microclima, densidad floral, tasa de visita floral, Chaetanthera, alta montaña, Andes, sistema reproductivo, cambio climático.

\section{ABSTRACT}

Insect pollinator activity can be influenced by biotic (e.g., patch floral density and floral display) or by abiotic factors (e.g., temperature, wind velocity, cloudiness). In spite of microsite, seasonal and interannual variation in temperature in the alpine zone, the consequences of local microclimatic variation on pollinator activity has been rarely studied in high mountain ecosystems. In this study we compared flower visitation rates on a northfacing slope and a west-facing slope in Chaetanthera apiculata $(3,100 \mathrm{~m}$ of altitude) and on an east-facing slope and a west-facing slope in Chaetanthera lycopodioides $(3,300 \mathrm{~m}$ of altitude). We studied the breeding system in each species in order to determine level of dependence on external pollinators. While the northfacing slope inhabited by $C$. apiculata was warmer $\left(1.8^{\circ} \mathrm{C}\right)$ and visited ( 7.8 -fold) more frequently than the west-facing slope, in C. lycopodioides the east-facing slope was warmer $\left(3{ }^{\circ} \mathrm{C}\right)$ and visited more frequently (4-fold) than the west-facing slope. In C. apiculata only Faunula leucoglene (Lepidóptera: Satyridae) showed higher activity in the warmer population. In $C$. lycopodioides, F. leucoglene and Liphantus sp. (Himenóptera: 
Andrenidae) showed higher activity rates in the warmer population. Flower visitation rate in Faunula leucoglene was correlated with temperature in $C$. apiculata and $C$. lycopodioides. Both $C$. apiculata and $C$. lycopodioides are partially self-compatible, and thus require exogenous pollen for maximum seed set. Our results show that in high elevation environments flower visitation rates can be highly variable and that microclimatic conditions can be more important for pollinator variation among populations than head density. Our results offer valuable insights on the potential consequences of global warming on plant pollination at high elevation.

Key words: microclimate, floral density, flower visitation rates, Chaetanthera, high mountains, Andes, breeding systems, global climate change.

\section{INTRODUCCIÓN}

Ha sido ampliamente documentado que la visita de los polinizadores a una planta puede ser afectada por factores bióticos como la densidad de flores en un parche (Schaal 1978, Thompson 1981, Schmitt 1983, Waser \& Price 1983, Sih \& Baltus 1987, Watkins \& Levin 1990) o el despliegue floral de las especies (e.g., Kannitz \& Maun 1991, Karron et al. 2004, Grindeland et al. 2005). No obstante, factores abióticos tales como las condiciones microclimáticas imperantes en el sitio donde se encuentran las plantas, también pueden influir fuertemente sobre el comportamiento y la actividad de los polinizadores (Herrera 1995a, 1995b, Rozzi et al. 1997). Los polinizadores son especialmente sensibles a cambios de temperatura, velocidad del viento y nubosidad.

El servicio de polinización es particularmente importante para las plantas de alta montaña. En una localidad en los Andes se ha encontrado altas proporciones de especies xenógamas, las cuales requieren obligadamente de vectores de polinización para producir semillas (Arroyo \& Squeo 1990). Consecuentemente, eventuales variaciones microclimáticas en estos ambientes cobran marcada importancia para el éxito reproductivo de las plantas con flor. No obstante, a diferencia de las variaciones altitudinales en la actividad de los insectos polinizadores, las cuales han sido relativamente bien estudiadas a nivel de poblaciones de una especie (Bingham \& Orthner 1998, Utelli \& Roy 2000, Totland \& Schulte-Herbrüggen 2003), y a nivel comunitario (Arroyo et al. 1985, Totland 1993), las consecuencias de las variaciones microclimáticas sobre las tasas de visita de los polinizadores han permanecido mucho menos estudiadas (Rozzi et al. 1997).

Los ambientes árticos y de alta montaña se caracterizan por presentar bajas temperaturas y fuertes vientos durante la estación de crecimiento (Mani 1962). Si bien los insectos pueden regular su temperatura corporal mediante mecanismos fisiológicos (endotermos) o a través de su comportamiento (ectotermos), la baja temperatura es una de las principales limitaciones de la actividad de los insectos en estos hábitats (Heinrich 1974, Heinrich \& Heinrich 1983, Watt 1968, May 1985). La endotermia requiere un alto suministro de energía (Heinrich 1974, Heinrich \& Heinrich 1983), por lo que en estos ambientes es más frecuente la polinización por insectos ectotermos (dípteros y lepidópteros), los cuales pueden forrajear con un bajo costo energético durante periodos esporádicos de calor (Arroyo et al. 1982). Lo anterior sugiere que variaciones microclimáticas producidas en cortas distancias podrían generar diferentes patrones de actividad de insectos polinizadores. No obstante, las respuestas funcionales de los distintos grupos de polinizadores frente a condiciones microclimáticas contrastantes han recibido poca atención. Por otra parte, no existen estudios en poblaciones naturales ( $\sin$ manipulación experimental) que hayan intentado evaluar conjuntamente cuál es el grado de importancia de las condiciones microclimáticas, la densidad de recursos florales y el tamaño floral para el éxito de la visita de polinizadores.

En la alta montaña de Chile central se ha establecido que laderas con exposición ecuatorial reciben más radiación solar directa por lo que mantienen mayores temperaturas del aire que laderas de exposición polar (Rozzi et al. 1989, Rozzi 1990). Rozzi (1990), y posteriormente Rozzi et al. (1997) encontraron que dichas variaciones microclimáticas están asociadas a cambios en los ensambles de polinizadores de Anarthrophyllum cumingii y Chuquiraga oppositifolia, lo cual puede afectar el flujo génico entre individuos que habitan en 
laderas de distinta exposición. No obstante, a pesar de que los estudios de Rozzi et al. (1990) y Rozzi et al. (1997) documentan claramente los efectos de las diferencias microclimáticas entre laderas de exposición norte y sur sobre la actividad de polinizadores, no existen más antecedentes acerca de los posibles efectos de diferencias microclimáticas entre pares de poblaciones de otras especies creciendo sobre laderas con otras exposiciones de manera de establecer el grado de generalidad del fenómeno. Los estudios de Rozzi et al. (1990) y Rozzi et al. (1997) fueron realizados en poblaciones ubicados en laderas ubicadas a los $2.500 \mathrm{~m}$ de altitud. Teniendo en cuenta que existen diferencias notables en los ensambles de polinizadores a esta altura en laderas de exposición opuesta, es esperable que dicho efecto sea aún mayor sobre los $3.000 \mathrm{~m}$ de altitud donde la temperatura promedio generalmente es menor.

En este estudio evaluamos las consecuencias de las diferencias microclimáticas, la densidad de flores en el parche y el tamaño floral, entre poblaciones ubicadas en laderas de distinta exposición solar sobre las tasas de visita de polinizadores. Específicamente nos planteamos las siguientes preguntas: (i) ¿En qué medida las condiciones microclimáticas determinan el éxito de la visita de polinizadores, independientemente de la densidad de flores y el tamaño de las mismas? (ii) ¿Cuán sensibles pueden ser los distintos grupos de polinizadores a dichas variables?

Se estudiaron dos especies del género Chaetanthera. En Chaetanthera apiculata (3.100 $\mathrm{m}$ de altitud) comparamos dos poblaciones, una creciendo sobre una ladera de exposición norte y otra creciendo en una ladera de exposición oeste. Esperamos que la ladera de exposición norte presente mayores temperaturas que la ladera oeste debido a que: (i) recibiría mayor incidencia diaria de los rayos solares; y (ii) la ladera de exposición oeste se encuentra más expuesta a vientos ascendentes que laderas de exposición norte. Para el caso de Chaetanthera lycopodioides (3.300 m de altitud) comparamos una población de exposición oeste y una población de exposición este. Esperamos que esta última población presente un microclima más benigno debido a que: (i) esta se encuentra en una condición de valle más cerrado y menos expuesto a los vientos ascendentes que la población de exposición oeste; y (ii) debido a que el sol sale por el oriente, la población este presenta un intervalo de luz solar diario mayor que la población oeste, alcanzando las temperaturas mínimas requeridas para el forrajeo más temprano que en la población oeste. Dado que en esta zona la actividad de los polinizadores está restringida al periodo entre las 10:00-17:00 hrs, la ventana temporal para la polinización es menor en la población oeste.

Dado que nuestro objetivo fue determinar el efecto de las variaciones microclimáticas sobre las diferencias entre poblaciones en las tasas de visita de insectos polinizadores en condiciones naturales, decidimos no realizar ningún tipo de manipulación de la densidad de floral en los parches. Debido a que las eventuales diferencias en las tasas de visita solo cobran relevancia si las plantas dependen del servicio de polinización, determinamos además el grado de compatibilidad genética de ambas especies.

\section{MATERIALES Y MÉTODOS}

\section{Especies de estudio}

El género Chaetanthera (Asteraceae: Mutisieae) es endémico del sur de Sudamérica (Cabrera 1937). De las 42 especies que lo componen, 36 se encuentran en Chile y están fuertemente concentradas en la zona de clima mediterráneo y de desierto con lluvias de invierno (Cabrera 1937). Hasta la fecha, todas las especies del género Chaetanthera estudiadas son entomófilas (Arroyo et al. 1982), aunque existen diferencias notables en las tasas de visita entre especies (e.g., Arroyo et al. 2006). Dentro de este género existe un amplio rango de variación en el tamaño de los capítulos y en los niveles de autocompatibilidad (Arroyo MTK, datos no publicados). Chaetanthera apiculata y $C$. lycopodioides son hierbas perennes que no superan los 3-4 y 6-8 $\mathrm{cm}$ de altura, respectivamente. Ambas especies presentan capítulos con flores tubulares de color amarillo (fértiles) y flores liguladas de color blanco (estériles para el sexo femenino). No existen mediciones de néctar disponibles para estas especies, sin embargo, las cantidades de néctar en ellas son tan pequeñas que son 
imperceptibles para el ojo humano. Mientras $C$. lycopodioides pertenece a la sección Oriastrum, $C$. apiculata pertenece a la sección Egania (sensu Cabrera 1937). Ambas secciones en conjunto forman un clado bien diferenciado de aquel que conforman las secciones Tylloma, Carmelita, Glandulosa, Proselia y Euchaetanthera (Hershkovitz et al. 2006).

\section{Sitio de estudio}

Este estudio fue realizado en la cordillera de los Andes de Chile central, en el sector de la Parva $\left(33^{\circ} 19^{\prime} \mathrm{S}, 70^{\circ} 16^{\prime} \mathrm{O}\right)$ y Valle Nevado

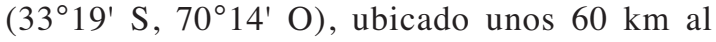
este de la ciudad de Santiago. En esta zona la vegetación se caracteriza por la dominancia de plantas en cojín (e.g., Laretia acaulis, Azorella spp. y Anarthrophyllum gayanum). Junto con ellas, son abundantes las hierbas perennes de tamaño mediano tales como: Phacelia secunda, Taraxacum officinale, Erigeron andicola, Haplopappus spp., Nassauvia spp., Perezia spp. y Senecio spp. (Cavieres et al. 2000).

Escogimos dos poblaciones de C. apiculata de altura equivalente ( $3.100 \mathrm{~m}$ de altitud) creciendo en laderas de exposiciones norte y oeste, separadas por unos $800 \mathrm{~m}$, denominadas API-N y API-O, respectivamente, las que fueron muestreadas durante febrero de 2005. Además, se estudiaron dos poblaciones de $C$. lycopodioides creciendo a aproximadamente
$3.300 \mathrm{~m}$ en laderas de exposición este y oeste. Estas poblaciones fueron muestreadas durante enero y febrero de 2004 (Tabla 1). En ambos casos, además de sus exposiciones solares, las poblaciones fueron seleccionadas debido a que presentaban densidades de plantas que posibilitaban la observación de varios individuos simultáneamente. En cada población advertimos varios parches de plantas, los cuales fueron elegidos en función de su representatividad de la población. A causa del número limitado de observadores, las dos poblaciones de una misma especie no fueron muestreadas en los mismos días, razón por la cual decidimos alternar el muestreo de las poblaciones. Además, con el propósito hacer válidas las comparaciones entre poblaciones, solo realizamos observaciones de polinizadores en días totalmente despejados.

\section{Condiciones microclimáticas}

Con el propósito de comparar las condiciones microclimáticas imperantes en cada una de las poblaciones muestreadas registramos la temperatura del aire a $20 \mathrm{~cm}$ del suelo cada 30 minutos, entre las 10:00 y las 16:30 h, durante todos los días que observamos polinizadores. Esto nos permitió conocer las temperaturas medias, máximas y mínimas, y su coeficiente de variación a lo largo de los tres días de observación y establecer las diferencias entre los microclimas de las diferentes laderas (Tabla 1).

TABLA 1

Condiciones microclimáticas de exposición, temperatura y altitud de las poblaciones estudiadas. Se indican las temperaturas: medias, máximas (máx), mínimas (mín), y su coeficiente de variación $(\mathrm{CV})$

Microclimatic conditions of solar exposure, ambient temperature, and altitude of studied populations. Mean, maximum $(\max )$, minimum $(\min )$ temperatures, and coefficient of variation $(\mathrm{CV})$ are shown

\begin{tabular}{lcccccrr}
\hline Especie & Código & Exposición & \multicolumn{3}{c}{ Temperatura $\left({ }^{\circ} \mathrm{C}\right)$} & Localidad & Altura \\
\cline { 4 - 6 } & & & Media & Máx - Mín & CV (\%) & \\
\hline C. apiculata & API-N & Norte & 18,7 & $14,0-24,0$ & 12,2 & La Parva & 3.160 \\
& API-O & Oeste & 16,9 & $13,0-21,0$ & 12,9 & La Parva & 3.100 \\
C. lycopodioides & LYC-E & Este & 20,7 & $17,0-24,0$ & 8,2 & Valle Nevado & 3.250 \\
& LYC-O & Oeste & 17,7 & $15,0-20,0$ & 8,4 & La Parva & 3.300 \\
\hline
\end{tabular}




\section{Sistema reproductivo}

Para determinar el sistema reproductivo se realizaron tres tratamientos: (i) polinización cruzada manual, en el cual las plantas recibieron polen de otros individuos conespecíficos distantes a lo menos a 10 metros para minimizar la posibilidad de endogamia biparental; (ii) autopolinización manual, que consistió en polinizar los capítulos con polen del mismo individuo; y (iii) autopolinización espontánea, que consistió en embolsar los capítulos florales al estado de yema floral para evaluar la producción autónoma de semillas (Kearns \& Inouye 1993). En general, un capítulo por planta fue tratado en $C$. lycopodioides. En $C$. apiculata el número de capítulos tratado por planta varió entre uno y dos. Para asegurar el éxito de los cruzamientos y autopolinizaciones estos fueron realizados al menos cinco veces. Los capítulos florales se mantuvieron embolsados hasta el final de la estación de crecimiento, cuando se contó el número de semillas y flores producidos por cada capítulo. Para determinar el sistema reproductivo estimamos el índice de autoincompatibilidad (IAI), mediante la razón entre el número de aquenios por capítulo obtenido en la prueba de autopolinización cruzada manual y el número de aquenios por capítulo obtenido en el tratamiento de polinización cruzada manual (Ruiz \& Arroyo 1978). Este índice va entre cero (autoincompatibilidad) y uno (autocompatibilidad). Además, comparamos la proporción de aquenios y frutos producidos por capítulo para cada tratamiento usando la prueba no paramétrica U de Mann-Whitney con la corrección de Bonferroni.

\section{Actividad de polinizadores}

Las observaciones de polinizadores en cada población fueron efectuadas siguiendo el procedimiento descrito en Utelli \& Roy (2000). Este consistió en ocho períodos diarios de 30 minutos de observación, efectuados simultáneamente por dos observadores en parches distintos de la misma población. Este procedimiento se repitió durante tres días, de manera que en cada población se completó un total de 48 periodos de observación de $30 \mathrm{~min}$ cada uno. En cada parche de observación, contabilizamos el número de capítulos florales abiertos y expresamos la tasa de visita floral de polinizadores como el número de visitas por capítulo floral por $30 \mathrm{~min}$. Comparamos las tasas de visita floral promedio de todo el ensamble de polinizadores y para los distintos órdenes de polinizadores, entre ambos pares de poblaciones. Posteriormente comparamos la tasa de visita especie por especie entre pares de laderas. Las observaciones de polinizadores se efectuaron en días completamente despejados. Consideramos que un capítulo fue exitosamente visitado cuando el insecto tocó algún florete del disco del capítulo floral. Posteriormente, capturamos e identificamos los insectos visitadores y verificamos la presencia de polen con una lupa.

\section{Rasgos florales y densidad de capítulos por parche}

Con el propósito de examinar los posibles efectos de la densidad de capítulos florales sobre la tasa de vista de polinizadores, cada vez que observamos polinizadores contabilizamos el número de capítulos abiertos en cada parche y su área, obteniendo así la densidad de capítulos en cada parche (número de capítulos $\mathrm{m}^{-2}$ ). Además, registramos los tamaños de los capítulos en todas las poblaciones estudiadas, para lo cual medimos el diámetro total y el diámetro del disco de 100 capítulos florales de cada población estudiada ( 1 por individuo).

\section{Análisis estadísticos}

La temperatura media del aire de los dos pares de poblaciones de cada especie de Chaetanthera fue comparada mediante pruebas t de Student. Las tasas de visita (variable dependiente) para las laderas de distinta exposición solar fueron comparadas a tres niveles: (i) entre pares de poblaciones de distinta exposición (factor), mediante ANCOVA en el cual la temperatura $\left({ }^{\circ} \mathrm{C}\right)$ y densidad de capítulos florales en el parche (capítulos $\mathrm{m}^{-2}$ ) fueron utilizadas como covariables (variables predictoras continuas). Las tasas de visita fueron normalizadas mediante raíz cuadrada del dato $+0,5$ (Utelli \& Roy 2000); (ii) entre diferentes órdenes de insectos polinizadores (Díptera, Himenóptera y Lepidóptera), mediante pruebas U de MannWhitney; y (iii) entre cada especie de insecto 
polinizador mediante pruebas de U de MannWhitney. Finalmente, el tamaño de los capítulos y la densidad de capítulos florales en el parche fueron comparados mediante pruebas t de Student.

\section{RESULTADOS}

\section{Condiciones microclimáticas}

En las poblaciones de Chaetanthera apiculata, la temperatura máxima y mínima del aire durante las observaciones varió entre $14-24{ }^{\circ} \mathrm{C}$ en la ladera norte y entre $13-21{ }^{\circ} \mathrm{C}$ en la ladera oeste (Tabla 1). La temperatura media en la ladera norte fue $1,8{ }^{\circ} \mathrm{C}$ mayor que en la oeste (prueba t de Student, $\mathrm{t}_{94}=-3,82 ; \mathrm{P}<0,001$ ). En el caso de $C$. lycopodioides las temperaturas variaron entre $17-24{ }^{\circ} \mathrm{C}$ en ladera este y entre 15-20 ${ }^{\circ} \mathrm{C}$ en ladera oeste (Tabla 1). La temperatura media de aire fue $3{ }^{\circ} \mathrm{C}$ mayor en la ladera este que en la oeste (prueba t de Student, $\left.\mathrm{t}_{86}=5,28 ; \mathrm{P}<0,001\right)$.

\section{Sistema de reproducción}

Ambas especies resultaron ser autocompatibles, presentando un sistema de reproducción mixto. En C. apiculata el índice de autocompatibilidad (ISI) fue de 0,56, mientras que en $C$. lycopodioides fue de 0,49 (Tabla 2). En $C$. apiculata la producción de semillas del tratamiento de polinización cruzada manual fue significativamente mayor que en el tratamiento de autopolinización manual, pero no en $C$. lycopodioides (Tabla 2). El nivel de autopolinización espontánea fue significativamente menor en comparación con la polinización cruzada en ambas especies, de manera que estas aumentarían su producción de semillas al ser polinizadas con polen exógeno.

\section{Actividad de polinizadores}

Chaetanthera apiculata fue visitada por 12 especies de insectos, de las cuales 7 visitaron API-N y 9 especies visitaron API-O, siendo solo Bombus terrestris (Hymenoptera: Apidae) y Faunula leucoglene (Lepidóptera: Satyridae) comunes a ambas poblaciones (Tabla 3). Los insectos polinizadores más abundantes de $C$. apiculata fueron $F$. leucoglene y Villa sp. 3 (Díptera: Bombyliidae).

No hubo diferencias en la hora de apertura de los capítulos florales de ambas poblaciones. Mientras el número total de visitas registrado en API-N fue de 1.004 (84\% del total de visitas), en API-O solo fue de 186 (16\% del total de visitas). Después de corregir por la densidad y temperatura, la tasa de visita (media $\pm 1 \mathrm{EE}$ ) de todo el ensamble de polinizadores fue 7,8 veces

TABLA 2

Tratamiento experimentales para detectar el sistema de reproducción de $C$. apiculata y $C$. lycopodioides. Los valores de porcentaje de aquenios por capítulo son promedios $\pm 2 \mathrm{EE}$

Experimental breeding system treatments in C.apiculata and C. lycopodioides. Values of percent of achenes formed per capitulum represent means \pm 2 SE

\begin{tabular}{lccc}
\hline Especies y tratamiento & Número de plantas & $\begin{array}{c}\text { Número de capítulos } \\
\text { (número de floretes) }\end{array}$ & Aquenios por capítulo* (\%) \\
\hline $\begin{array}{l}\text { Chaetanthera apiculata } \\
\text { Polinización cruzada manual }\end{array}$ & 27 & & \\
Autopolinización manual & 25 & $43(992)$ & $31,2^{2 \pm 5,5^{a}}$ \\
Autopolinización espontánea & 26 & $40(942)$ & $17,5^{a} \pm 5,5^{b}$ \\
Chaetanthera lycopodioides & & $40(919)$ & $3,7^{b} \pm 2,0^{c}$ \\
$\quad$ Polinización cruzada manual & 18 & $18(257)$ & $52,5 \pm 18,9^{a}$ \\
Autopolinización manual & 19 & $20(297)$ & $25,5^{a} \pm 13,9^{a b}$ \\
Autopolinización espontánea & 17 & $17(252)$ & $11,3 \pm 11,4^{b}$ \\
\hline
\end{tabular}

* Letras distintas indican diferencias significativas entre los tratamientos de polinización según la prueba U de MannWhitney con corrección de Bonferroni 
mayor en API-N $(0,446 \pm 0,031)$ que en API-O $(0,057 \pm 0,031)$, siendo esta diferencia estadísticamente significativa $\left(\mathrm{F}_{1,92}=57,97 ; \mathrm{P}<\right.$ 0,0001 ) (Fig. 1). Al comparar los distintos órdenes de polinizadores solo encontramos diferencias entre poblaciones para lepidópteros $(\mathrm{U}=58,5 ; \mathrm{P}<0,001)$. Cuando comparamos la tasa de visita de cada especie de polinizador entre ambas laderas, solo Faunula leucoglene presentó una actividad significativamente mayor en API-N (Fig. 2, Tabla 3).
Tanto la densidad de capítulos florales en los parches como el tamaño floral fueron significativamente mayores en API-O que en API-N (Tabla 4). La tasa de visita de Faunula leucoglene se correlacionó positivamente con la temperatura $(\mathrm{r}=0,46 ; \mathrm{P}<0,0001)$ y negativamente con la densidad de capítulos florales $(\mathrm{r}=-0,51 ; \mathrm{P}<0,0001)$.

Chaetanthera lycopodioides fue visitada por un total de 16 especies de insectos (Tabla 3). De ellas, 14 visitaron LYC-E y nueve

TABLA 3

Lista de polinizadores y sus tasas de visita floral por especie (número de visitas por capítulo floral por $30 \mathrm{~min}$ ). Las tasas de visita de cada especie de polinizador fueron comparadas mediante una prueba U de Mann-Whitney

List of pollinators and their floral visitation rates (number of visits per flower head per $30 \mathrm{~min}$ ). Visitation rates of each pollinator were compared using the Mann-Whitney U-test

\begin{tabular}{|c|c|c|c|c|c|c|c|}
\hline \multirow[t]{2}{*}{ Orden/Familia } & \multirow[t]{2}{*}{ Especie } & \multicolumn{3}{|c|}{ C. apiculata } & \multicolumn{3}{|c|}{ C. lycopodioides } \\
\hline & & Norte & Oeste & Valor de Z & Este & Oeste & Valor de Z \\
\hline \multicolumn{8}{|l|}{ Diptera } \\
\hline \multirow[t]{8}{*}{ Bombyliidae } & Villa semifuscata & & & & 0,0064 & $<0,0010$ & 0,01 \\
\hline & Villa flavicura & & & & 0,0122 & 0,0176 & $-1,11$ \\
\hline & Villa sp. 3 & 0,0104 & - & $-1,05$ & 0,0030 & 0,019 & $-1,28$ \\
\hline & Villa gayi & - & 0,0012 & 0,57 & & & \\
\hline & Diplocampta sp. & 0,0096 & - & $-0,35$ & 0,0010 & - & 0,01 \\
\hline & Sericosoma irwini & - & $<0,0010$ & 0,17 & 0,0040 & - & 0,18 \\
\hline & Lyophlaeba canescens & & & & 0,0010 & $<0,0010$ & 0,18 \\
\hline & Scaeva melanostoma & - & $<0,0010$ & 0,17 & 0,0046 & $<0,0005$ & $-0,51$ \\
\hline Tachiniidae & No identificado & & & & 0,0053 & 0,0089 & $-1,16$ \\
\hline Nemestrinidae & No recolectado & - & 0,0018 & 0,17 & & & \\
\hline \multicolumn{8}{|l|}{ Himenoptera } \\
\hline Apidae & Bombus terrestris & $<0,0010$ & $<0,0010$ & $-0,00$ & & & \\
\hline Megachilidae & Anthidium spinosai & - & $<0,0010$ & 0,17 & & & \\
\hline Andrenidae & Liphantus sp. & & & & 0,2267 & 0,0490 & $3,87 * * *$ \\
\hline \multicolumn{8}{|l|}{ Lepidoptera } \\
\hline Satyridae & Faunula leucoglene & 0,4331 & 0,0356 & $-8,0 * * *$ & 0,0673 & - & $5,27 * * *$ \\
\hline \multirow[t]{2}{*}{ Nymphalidae } & Yramea lathonioides & 0,0018 & - & $-1,05$ & $<0,0005$ & - & 0,18 \\
\hline & Vanessa terpsichore & - & 0,0004 & 0,17 & & & \\
\hline Hesperiidae & Hylephila fasciolata & $<0,0010$ & - & $-0,17$ & 0,044 & - & 0,70 \\
\hline Pieridae & Phulia nymphula & $<0,0010$ & - & 0,17 & $<0,0010$ & - & 0,18 \\
\hline Lycaenidae & Pseudolucia chileis & & & & 0,0013 & 0,0066 & 0,18 \\
\hline \multicolumn{8}{|l|}{ Coleoptera } \\
\hline Melyridae & Arthrobrachus sp. & & & & 0,0029 & - & 0,53 \\
\hline No identificado & No recolectado & - & $<0,0010$ & 0,17 & - & 0,0020 & 0,18 \\
\hline \multicolumn{2}{|c|}{ Número total de visitas } & 1002 & 184 & & 597 & 212 & \\
\hline \multicolumn{2}{|c|}{ Número total de especies } & 7 & 9 & & 15 & 9 & \\
\hline
\end{tabular}

$(* * *)=\mathrm{P}<0,01$ 
especies visitaron LYC-O, siendo 8 de ellas comunes a ambas poblaciones (Tabla 3). Los principales insectos polinizadores de $C$. lycopodioides fueron Liphantus sp. (Hymenóptera: Andrenidae), Faunula leucoglene y Villa sp.3

A diferencia de $C$. apiculata encontramos que los capítulos florales de LYC-O se abrieron media hora más tarde que en LYC-E. Esta última población recibió un total de 578 visitas (73\% del total de visitas), mientras que LYC-O solamente recibió 209 visitas (27\% del total de visitas). Luego de corregir por la temperatura y la densidad de capítulos en los parches, la tasa de visita de todo el ensamble de polinizadores fue cuatro veces mayor en LYC-E $(0,307 \pm$ $0,040)$ que en LYC-O $(0,075 \pm 0,040)\left(\mathrm{F}_{1,84}=\right.$ 15,$85 ; \mathrm{P}<0,0001$ ) (Fig. 1).

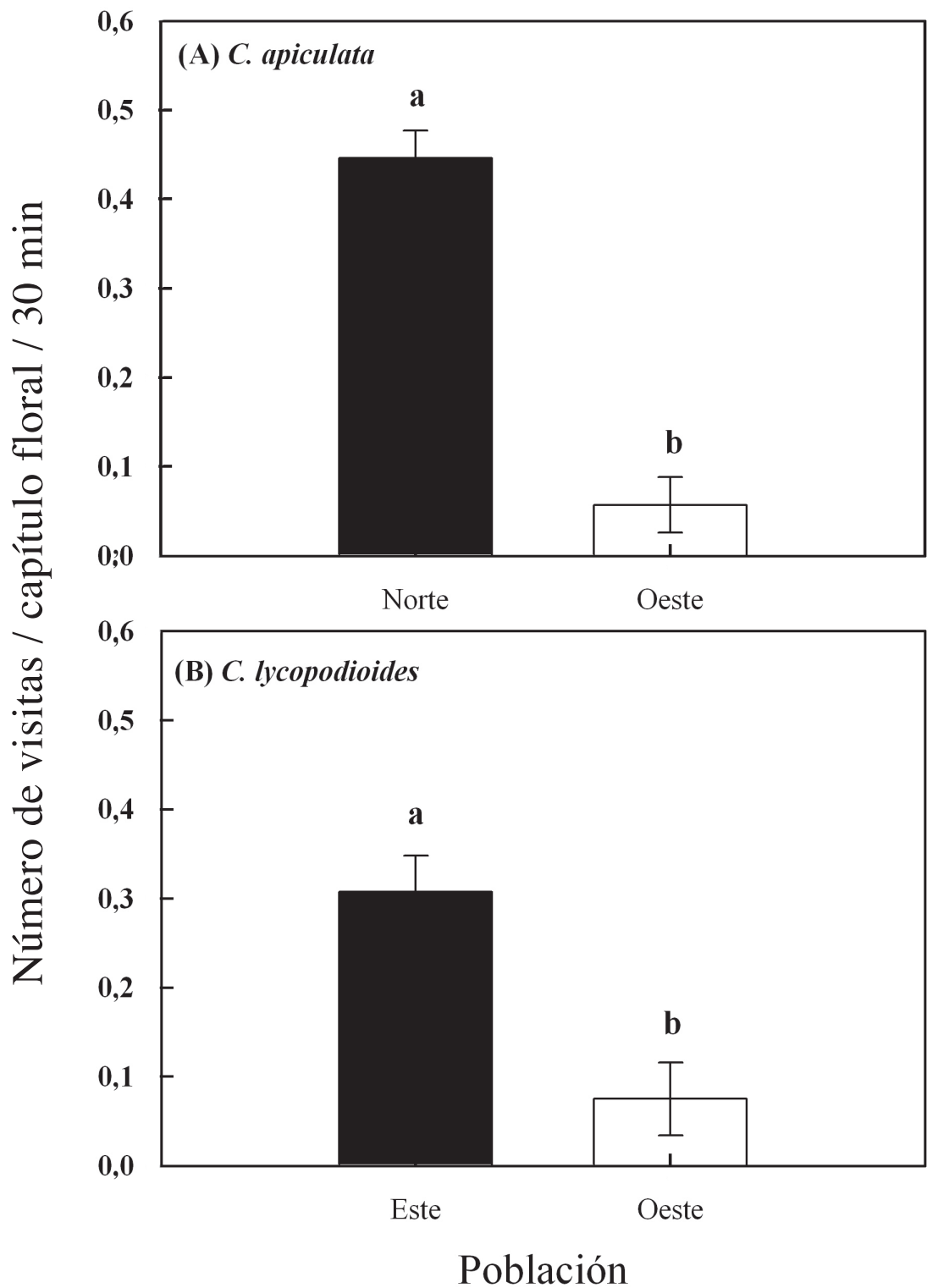

Fig. 1: Tasas de visita floral promedio por polinizadores $( \pm 1 \mathrm{EE})$ en poblaciones de distinta exposición para (A) C. apiculata y (B) C. lycopodioides. Letras diferentes indican diferencias estadísticamente significativas con $\mathrm{P}<0,05$.

Mean floral visitation rates by pollinators $( \pm 1 \mathrm{SE}$ ) on different facing slopes for (A) C. apiculata and (B) C. lycopodioides. Different letters are used to indicate statistically significant differences at $\mathrm{P}<0.05$. 
Al comparar la tasa de visita de los distintos órdenes de polinizadores entre poblaciones de distinta exposición, solo se encontraron diferencias para los lepidópteros $(\mathrm{U}=432, \mathrm{P}<$ $0,0001)$ y para los himenópteros $(\mathrm{U}=641,5, \mathrm{P}$ $=0,0001)$. Al analizar la tasa de visita de cada especie de polinizador, encontramos que solo
Liphantus sp. (Himenóptera: Andrenidae) y $F$. leucoglene presentaron actividades significativamente mayores en LYC-E que en LYC-O (Fig. 2, Tabla 3).

El tamaño de los capítulos florales fue significativamente mayor en LYC-E que en LYC-O (Tabla 4). Por el contrario, la densidad

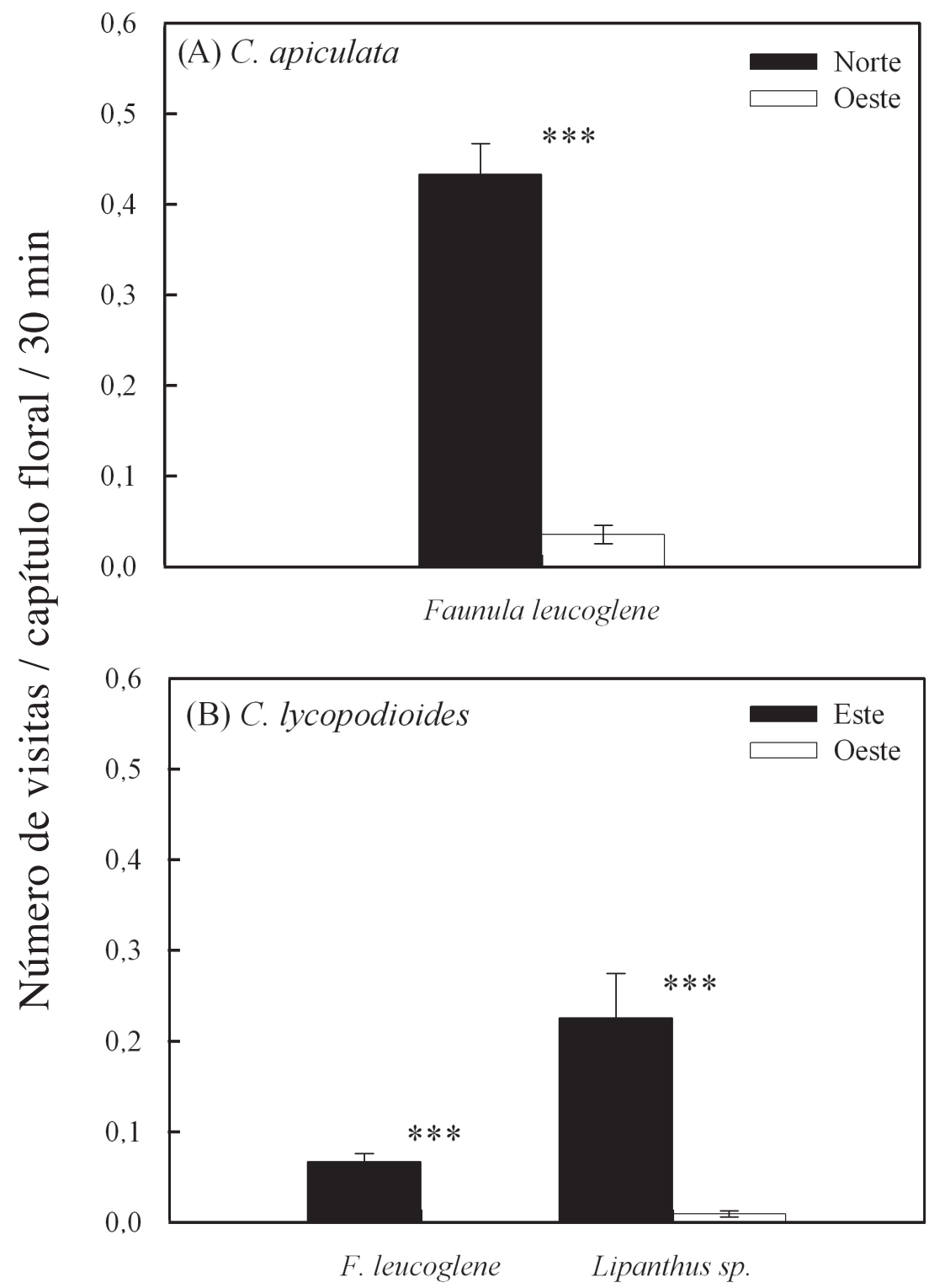

\section{Especie}

Fig. 2: Tasas de visita floral promedio $( \pm 1 \mathrm{EE})$ para las especies de polinizadores que difirieron entre pares de laderas con diferentes microclimas: (A) C. apiculata y (B) C. lycopodioides; $(* * *)=$ $\mathrm{P}<0,001$.

Mean floral visitation rates $( \pm 1 \mathrm{SE})$ for the pollinator species that showed differences among slopes with different microclimates: (A) C. apiculata and (B) C.lycopodioides; $(* * *)=\mathrm{P}<0.001$. 
TABLA 4

Comparación de los rasgos florales y de la densidad de capítulos (número de capítulos $\mathrm{m}^{-2}$ ) entre laderas de distinta exposición. Los valores son promedios $\pm 2 \mathrm{EE}$

Comparison of the floral traits and density of capitula (number of heads $\mathrm{m}^{-2}$ ) between different facing slopes pairs. Values are means $\pm 2 \mathrm{SE}$

\begin{tabular}{|c|c|c|c|c|c|c|}
\hline \multirow[t]{2}{*}{ Rasgo } & \multicolumn{3}{|c|}{ C. apiculata } & \multicolumn{3}{|c|}{ C. lycopodioides } \\
\hline & Norte & Oeste & Valor de $\mathrm{t}$ & Este & Oeste & Valor de $\mathrm{t}$ \\
\hline Diámetro del capítulo & $18,5 \pm 0,40$ & $19,6 \pm 0,48$ & $3,5 *$ & $15,5 \pm 0,32$ & $13,8 \pm 0,34$ & $-3,8 *$ \\
\hline Diámetro del disco & $5,0 \pm 0,12$ & $5,4 \pm 0,16$ & $3,1^{*}$ & $3,3 \pm 0,10$ & $3,0 \pm 0,04$ & $-2,97 *$ \\
\hline Número de capítulos $\mathrm{m}^{-2}$ & $8,5 \pm 0,84$ & $14,1 \pm 0,84$ & $9,4 *$ & $15,3 \pm 3,8$ & $25,6 \pm 4,30$ & $5,3^{*}$ \\
\hline
\end{tabular}

(*) $\mathrm{P}<0,005$

de capítulos florales en los parches fue mayor en LYC-O. Mientras la tasa de visita de $F$. leucoglene solo se asoció positivamente con la temperatura $(\mathrm{r}=0,31 ; \mathrm{P}=0,003)$, la tasa de visita de Liphantus sp. no se asoció con la temperatura ni con la densidad de capítulos florales.

\section{DISCUSIÓN}

Nuestros resultados muestran que las diferencias microclimáticas locales pueden influir fuertemente sobre las tasas de visitas y la composición de especies de insectos polinizadores asociadas a poblaciones de diferente exposición solar en los Andes de Chile central. Junto con esto, nuestros resultados muestran que para ciertas especies de polinizadores, las condiciones microclimáticas son incluso más importantes que la densidad de recursos florales en los parches. En su conjunto, nuestros resultados son consistentes con el único estudio realizado en los Andes de Chile central por Rozzi et al. (1997), quienes también encontraron marcadas diferencias entre poblaciones en los patrones de visita de insectos sobre Anarthrophyllum cumingii y Chuquiraga opposifolia de distinta exposición solar.

La unidad de observación para calcular las tasas de visita en este estudio fue el capítulo floral, ello dado que el capítulo es funcionalmente equivalente a la flor de otras familias de plantas en cuanto a la atracción hacia los polinizadores. Resulta interesante entonces comparar los resultados con la tendencias comunitarias para el área de estudio publicado por Arroyo et al. (1985), en donde se presenta los promedios de las tasas de visita al nivel comunitario basado en el llamado "random walk", que es una metodología extensiva diseñada para muestrear las comunidades de acuerdo con las abundancias de las especies de plantas, moviéndose constantemente entre diferentes especies y poblaciones sin elegir densidades mayores. Las tasas de visitas obtenidas en $C$. lycopodioides y $C$. apiculata son altas comparadas con la tendencia comunitaria considerando la misma elevación. Al nivel comunitario las tasas de visita promedio obtenidas por Arroyo et al. (1985) equivalen a $0,205,0,125$ y 0,095 visitas por flor por $30 \mathrm{~min}$ para elevaciones bajas (2.200-2.600 $\mathrm{m}$ de altitud), medias (2.700$3.100 \mathrm{~m}$ de altitud) y altas (3.200-3.600 m de altitud), respectivamente; nosotros encontramos que las poblaciones de microclima más benigno de $C$. apiculata y $C$. lycopodioides tuvieron tasas mayores a las esperables considerando su altitud $\left(0,446\right.$ y 0,307 visitas $30 \mathrm{~min}^{-1}$, respectivamente). Sin embargo, en Chaetanthera euphrasioides, para la misma localidad y altura del estudio actual, las tasas de visita son dos órdenes de magnitud menores con respecto a la tendencia comunitaria (Arroyo et al. 2006). Los resultados para las tres especies de Chaetanthera en una misma localidad y altura demuestran que existe mucha variación alrededor de la tendencia general en la comunidad, incluso en un mismo género. Dichas diferencias son esperables, al comparar especies xenógamas, como $C$. apiculata y $C$. lycopodioides, con especies altamente 
autógamas como $C$. euphrasioides, y pueden esperarse para otros géneros de plantas en la zona. Desde luego, las poblaciones de $C$. lycopodioides y $C$. apiculata estudiadas fueron en promedio muy densas, por lo que las tasas probablemente son algo exageradas con respecto a la tendencia comunitaria en donde no se eligió densidades altas. Por otra parte, han transcurrido más de 20 años desde la fecha en que se efectuaron los muestreos comunitarios, de tal forma que es posible que la temperatura actual sea mayor que la publicada en Arroyo et al. (1985). La magnitud de las tasas de visita en las poblaciones de exposición norte de $C$. apiculata y de exposición este de $C$. lycopodioides resultaron ser altas y similares a las tasas de visita de poblaciones de Chaetanthera linearis y Chaetanthera flabellata, ambas especies del matorral subandino que crecen entre 900 y 1.100 m más abajo, donde las temperaturas son mucho mayores $\left(5-7{ }^{\circ} \mathrm{C}\right)$ (C. Torres-Díaz, resultados no publicados). Arroyo et al. (2006) propusieron que las tasas de visitas florales de especies xenógamas en la zonas alpinas no deberían variar con la altura, dado la existencia de mecanismos de compensación, como flores más atractivas, y longevidad floral mayor. Si bien no se trata de las mismas especies, este principio puede aplicarse a especies del mismo género con un sistema de reproducción similar, de modo que la igualdad de las tasas de visita en $C$. linearis y $C$. flabellata (ambas xenógamas) con $C$. apiculata y $C$. lycopodioides no resulta sorprendente.

Existen numerosos ejemplos de que, para especies de plantas dependientes de polinizadores, un incremento en la tasa de visita de insectos polinizadores puede producir aumentos del éxito reproductivo (e.g., Waser \& Price 1983, Sih \& Baltus 1987, Andersson 1996, Kunin 1997, Kirchner et al. 2005). Considerando que tanto la eficiencia (Herrera 1987), como el comportamiento de vuelo de los polinizadores es altamente variable entre grupos (Schmitt 1980), las diferencias interpoblacionales en los ensambles de polinizadores descritas aquí podrían determinar disparidad en la producción de semillas. Si bien, en términos genéticos, las dos especies de Chaetanthera son parcialmente autocompatibles, ellas incrementarían su desempeño reproductivo cuando reciben polen de individuos coespecíficos, lo cual sugiere que las diferencias en las tasas de visita entre poblaciones podrían tener repercusiones sobre el éxito reproductivo de las plantas. Sin embargo, en la práctica no fue posible medir el éxito reproductivo de ambas especies debido a los altos niveles de depredación de semillas predispersión realizada por larvas de lepidópteros (C. Torres-Díaz, observación personal). Esto sugiere que el éxito reproductivo final de poblaciones que crecen en laderas contrastantes, no solo dependerá del servicio de polinización, sino que también dependerá de los efectos de factores bióticos y abióticos sobre otras fases del ciclo de vida, como por ejemplo, la depredación de semillas (pre y posdispersión), el establecimiento y supervivencia de plántulas (Rozzi 1990).

\section{Microclima y actividad de insectos polinizadores}

Se ha documentado que las variaciones microclimáticas pueden determinar las diferencias en la composición de polinizadores asociados a plantas individuales (Herrera 1995b) o poblaciones de plantas (Rozzi et al. 1997). En ambientes de alta montaña, dípteros y lepidópteros son los visitadores florales predominantes (Mani 1962, Moldenke 1972, Primack 1978, Arroyo et al. 1982, Warren et al. 1988, Kearns 1992, Totland 1993). En términos generales, los insectos ectotermos son principalmente dípteros y lepidópteros, mientras que dentro de los endotermos predominan los himenópteros, existiendo también algunos dípteros y lepidópteros (Heinrich 1975). En ambas especies de Chaetanthera la tasa de visita de F. leucoglene (Lepidóptera) mostró una asociación positiva con la temperatura, a pesar de que la densidad de capítulos florales en los parches (y el tamaño de los capítulos florales en $C$. apiculata) fueron mayores en las poblaciones con microclimas menos benignos. Este hecho indica que para $F$. leucoglene las condiciones microclimáticas pueden influir más fuertemente sobre la variación en la actividad de polinizadores entre poblaciones que la disponibilidad de recursos florales. La actividad exclusiva de $F$. leucoglene en la población más cálida de $C$. lycopodioides encuentra su explicación en la biología de los lepidópteros. Dada la alta capacidad de vuelo 
de los lepidópteros (Schmitt 1980), en comparación con los dípteros y ciertos himenópteros, es probable que $F$. leucoglene concentre su actividad en sectores con microclima más benignos, lo cual explicaría la ausencia de visitas en la población de exposición oeste. En ambientes fríos los lepidópteros ectotérmicos pueden incrementar su temperatura corporal realizando detenciones para asolearse y calentarse en el suelo (Kingsolver 1985, 1987). Nosotros observamos que $F$. leucoglene se asolea lateralmente con las alas plegadas, comportamiento que fue principalmente observado en la población más fría. Se ha documentado que el desempeño termorregulatorio de los lepidópteros de climas fríos mejora con la cantidad de melanina, incrementando la capacidad de absorción de radiación solar para el calentamiento corporal (Watt 1968). Dada su coloración casi totalmente negra, F. leucoglene probablemente es uno de las lepidópteros mejor adaptado para la vida en la zona de estudio. En este sentido, es notable que los dípteros prácticamente desaparecen como polinizadores en poblaciones de $C$. lycopodioides a los $3.450 \mathrm{~m}$ de altitud en Valle Nevado. En el límite de distribución altitudinal de $C$. lycopodioides, los polinizadores son 6 especies de mariposas, entre las cuales la más importante es $F$. leucoglene (Arroyo et al. 2007). La diferencia entre los polinizadores en $C$. lycopodioides entre localidades separadas por $150 \mathrm{~m} \mathrm{de}$ altitud, nuevamente destaca la variabilidad espacial de los ensambles de polinizadores a las mayores alturas en zonas alpinas.

Por otro lado, a pesar de que no encontramos una asociación positiva entre la temperatura y la tasa de visita de Liphantus sp., la mayor actividad de esta especie en la población más cálida sugiere cierto efecto de la temperatura. Aunque los himenópteros son por lo general endotermos, se ha documentado que la capacidad de termorregulación de los himenópteros decrece a medida que el tamaño corporal disminuye (Bishop \& Armbruster 1999). Liphantus sp. es un himenóptero de tamaño pequeño $(\sim 2-3 \mathrm{~mm})$, que solo realiza vuelos a cortas distancias (1-3 m) y cuyas poblaciones son muy locales, teniendo probablemente ámbitos de hogar restringidos. Esto indica que las poblaciones de Liphantus sp. podrían tener cierta tendencia a asociarse a lugares con microclimas más benignos, pudiendo presentar tamaños poblacionales mayores en laderas cálidas.

\section{Implicancias para el calentamiento global}

Se ha estimado que la temperatura del planeta ha aumentado en $1{ }^{\circ} \mathrm{C}$ (Walther 2003), y ciertos modelos han proyectado aumentos de $1,8-4{ }^{\circ} \mathrm{C}$ por sobre la temperatura promedio actual en este siglo (IPCC 2001). Mientras diversos autores han estudiado experimentalmente las consecuencias del calentamiento global (e.g., Henry \& Molau 1997, Price \& Waser 1998, Hobbie et al. 1999), mucho del conocimiento debiese provenir de la observación de la variación natural de las poblaciones a escala de paisaje (Price \& Waser 1998). En este contexto, nuestros resultados cobran relevancia en un escenario de calentamiento global. Las diferencias en la temperatura promedio entre laderas de exposición opuestas fueron de $1,8{ }^{\circ} \mathrm{C}$ (C. apiculata) y $3,0{ }^{\circ} \mathrm{C}(C$. lycopodioides $), 10$ cual está dentro del rango de incremento en la temperatura propuesto para este siglo.

Nuestros resultados muestran que algunas especies de insectos aumentan su actividad en las laderas cálidas con respecto a las laderas frías, lo cual demuestra que en las laderas de exposición oeste ciertas especies están por debajo de su temperatura óptima para el forrajeo. Por lo tanto, posiblemente los futuros aumentos en la temperatura del planeta provocarían un incremento de la actividad de polinizadores en las poblaciones sujetas a microclimas más fríos. Por otro lado, si después de un aumento de la temperatura del planeta de $1,8-4{ }^{\circ} \mathrm{C}$ las laderas de exposición norte y este están por sobre la temperatura óptima de forrajeo de los insectos, sus tasas de visita debiesen disminuir. Si por el contrario, las temperaturas aún están por debajo del óptimo. las tasas de visita podrían seguir aumentando. No obstante, las predicciones anteriores son también dependientes de aspectos biológicos relacionados con la biología de los insectos, haciéndose necesario conocer cómo responderá la biología del desarrollo de los insectos y los tamaños poblacionales ante el aumento de la temperatura.

En conclusión, nuestros resultados indican que desde la perspectiva de la polinización, los ambientes de alta montaña están lejos de ser 
ambientes homogéneos, correspondiendo más bien a mosaicos altamente variables y heterogéneos. El significado de este tipo de variación para la evolución de los sistemas de reproducción y la morfología floral de las plantas de alta montaña aún no se conoce cabalmente.

\section{AGRADECIMIENTOS}

Agradecemos a Karen Balboa, Maritza Mihoč, Susana Gómez-González, Carol Peña y Patricio Torres-Morales por su valiosa ayuda en terreno. Cristián Torres-Díaz ha sido becario MECESUP (UCO 9906) y becario del Milenio (P02-051-F). Esta investigación fue financiada por FONDECYT (1020956, 7020956), ECOSFrancia-Chile C01B03, el contrato P02-051 ICM to the Center for Advanced Studies in Ecology and Biodiversity (CMEB), que actualmente es el Instituto de Ecología y Biodiversidad (IEB). Agradecemos a los centros invernales de La Parva y Valle Nevado por todas las facilidades prestadas en terreno.

\section{LITERATURA CITADA}

ANDERSSON S (1996) Floral display and pollination success in Senecio jacobaea (Asteraceae): Interactive effects of heads and corymb size. American Journal of Botany 83: 71-75.

ARROYO MTK, R PRIMACK \& JJ ARMESTO (1982) Community studies in pollination ecology in the high temperate Andes of central Chile I. Pollination mechanisms and altitudinal variation. American Journal of Botany 69: 82-97.

ARROYO MTK, JJ ARMESTO \& R PRIMACK (1985) Community studies in pollination ecology in the high temperate Andes of central Chile. II. Effect of temperature on visitation rates and pollination possibilities. Plant Systematic and Evolution 149: 187-203.

ARROYO MTK \& F SQUEO (1990) Relationships between plant breeding and pollination. En: Kawano $S$ (ed) Biological approaches and evolutionary trends in plants: 205-227. Academy Press, London, United Kingdom.

ARROYO MTK, R ROZZI, F SQUEO \& E BELMONTE (1990) Pollination in tropical and temperate high elevation ecosystems: hypotheses and the Asteraceae as a test case. En: Winiger M, Weismann U \& J Rhekert (eds) Mounts Kenya area: differentiation and dynamics of a tropical mountain ecosystem: 21-31. African Studies Series A8, University of Nairobi, Berne, Switzerland

ARROYO MTK, MS MUÑOZ, C HENRÍQUEZ, I TILLBOTTRAUD \& F PÉREZ (2006) Erratic pollination, high selfing levels and their correlates and consequences in an altitudinally widespread above-tree-line species in the high Andes of Chile. Acta Oecologica 30: 248-257.

ARROYO MTK, UD TILL-BOTTRA I, C TORRESDÍAZ, CA HENRÍQUEZ, \& J MARTÍNEZ (2007) Display size preferences and foraging habits of high Andean butterflies pollinating Chaetanthera lycopodioides (Asteraceae) in the subnival of the central Chilean Andes. Arctic, Antarctic and Alpine Research 39: 347-352.

BINGHAM RA \& AR ORTHNER (1998) Efficient pollinators of alpine plants. Nature: 391: 238-239.

BISHOP JA \& WS ARMBRUSTER (1999) Thermoregulatory abilities of Alaskan bees: effects of size, phylogeny and ecology. Functional Ecology 13: 711-724.

CABRERA AL (1937) Revisión de género Chaetanthera (Compositae). Revista del Museo de La Plata, Sección Botánica (Argentina) 1: 87-120.

CAVIERES L, A PEÑALOZA \& MTK ARROYO (2000) Altitudinal vegetation belts in the high-Andes of central Chile $\left(33^{\circ} \mathrm{S}\right)$. Revista Chilena de Historia Natural 73: 331-344.

GRINDELAND JM, N SLETVOLD \& RA IMS (2005) Effects of floral display size and plant density on pollinator visitation rate in a natural population of Digitalis purpurea. Functional Ecology 19: 383-390.

HEINRICH B (1974) Thermoregulation in endothermic insects. Science 185: 747-755.

HEINRICH B (1975) Energetics of pollination. Annual Review of Ecology and Systematics 6: 139-169.

HEINRICH B \& MJE HEINRICH (1983) Size and caste in temperature regulation by bumblebees. Physiological Zoology 56: 552-562.

HENRY GHR \& U MOLAU (1997) Tundra plants and climate changes: the international tundra experiments (ITEX). Global Change Biology: 3: 1-9.

HERRERA CM (1987) Components of pollinator "quality": comparative analysis of a diverse insect assemblage. Oikos 50: 79-90.

HERRERA CM (1995a) Floral biology, microclimate and pollination by ectothermic bees in an earlyblooming herb. Ecology 76: 218-228.

HERRER A CM (1995b) Microclimate and individual variation in pollinators: flowering plants are more than their flowers. Ecology 76: 1516-1524.

HERSHKOVITZ M, MTK ARROYO, C BELL \& LF HINOJOSA (2006) Phylogeny of Chaetanthera (Asteraceae: Mutisieae) reveals both ancient and recent origins of the high elevation lineages. Molecular Phylogenetics and Evolution 41: 594605.

HOBBIE SE, A SHEVTSOVA \& FS CHAPIN (1999 Plant responses to species removal and experimental warming in Alaskan tussock tundra. Oikos 84: 417-434

IPCC (2001) International panel on climate change: climate change 2001 - impacts, adaptation, and vulnerability. Cambridge University Press, Cambridge, United Kingdom.

KANNITZ PG \& MA MAUN (1991) An experimental study of floral display size and reproductive success in Vivurnum opulus: the importance of grouping. Canadian Journal of Botany 69: 394-399.

KARRON JD, RJ MITCHELL, HOLMQUIST KG, JM BELL \& B FUNK (2004) The influence of floral display size on selfing rates in Mimulus ringens. Heredity 92: 242-248.

KEARNS CA (1992) Anthophilous fly distribution across an elevation gradient. American Midland Naturalist 127: $172-182$. 
KEARNS CA \& DW INOUYE (1993) Techniques for pollination biologists. University Press of Colorado, Boulder, Colorado, USA. 583 pp.

KINSOLVER JG (1985) Butterfly thermoregulation: organismic mechanisms and population consequences. Journal of Research on the Lepidopterans 24: 1-20.

KIRCHNER F, SH LUIJTEN, E IMBERT, M RIBA, M MAYOL, SC GONZÁLEZ-MARTÍNEZ, A MIGNOT \& B COLAS (2005) Effects of local density on insect visitation and fertilization success in the narrow-endemic Centaurea corymbosa (Asteraceae). Oikos 111: 130-142.

KUNIN WE (1997) Population size and density effects in pollination: pollinator foraging and plant reproductive success in experimental arrays of Brassica Kaber. Journal of Ecology 85: 225-234.

MANI MS (1962) Introduction to high altitude entomology: insect life above the timberline in the north-western Himalayas. Methuen, London, United Kingdom.

MOLDENKE AR \& PG LINCOLN (1972) Pollination ecology in montane Colorado. Phytologia 42: 349379.

MAY ML (1985) Thermoregulation. En: Kerkut GA \& LI Gilbert (eds) Comprehensive insect physiology, biochemistry and pharmacology: 507-552. Pergamon Press, Oxford, United Kingdom.

PRICE MV \& NM WASER (1998) Effects of experimental warming on plant reproductive phenology in a subalpine meadow. Ecology 94: 1261-1271.

PRIMACK RB (1978) Variability in New Zealand montane and alpine pollinator assemblages. New Zealand Journal of Ecology 1: 66-73.

ROZZI R, JD MOLINA \& P MIRANDA (1989) Microclima y períodos de floración en laderas de exposición ecuatorial y polar en los Andes de Chile central. Revista Chilena de Historia Natural 62: 7584.

ROZZI R (1990) Periodos de floración y especies de polinizadores en poblaciones de Anarthrophyllum gayanum y Chuquiraga oppositifolia que crecen sobre laderas de exposición norte y sur. Tesis de Magíster, Facultad de Ciencias, Universidad de Chile, Santiago, Chile. 125 pp.

ROZZI R, MTK ARROYO \& JJ ARMESTO (1997) Ecological factors affecting gene flow between populations of Anarthrophyllum cumningii (Papilionaceae) growing on equatorial- and polarfacing slopes in the Andes of central Chile. Plant Ecology 132: 171-179.
RUIZ T \& MTK ARROYO (1978) Plant reproductive ecology of a secondary deciduous forest in Venezuela. Biotropica 10: 221-230.

SCHAAL BA (1978) Density dependent foraging on Liatris pychnostachya. Evolution 32: 452-454.

SCHMITT J (1980) Pollinator foraging behaviour and gene dispersal in Senecio (Compositae). Evolution 34: 934-943.

SCHMITT J (1983) Flowering plant density and pollinator visitation in Senecio. Oecologia 60: 97-102.

SIH A \& MS BALTUS (1987) Patch size, pollinator behaviour, and pollinator limitation in catnip. Ecology 68: 1679-1690.

THOMPSON JD (1981) Spatial and temporal components of resource assessment by flower-feeding insects. Journal of Animal Ecology 50: 49-59.

TOTLAND $\varnothing \&$ B SCHULTE-HERBRÜGGEN (2003) Breeding system, insect flower visitation, and floral traits of two Alpine Cerastium Species in Norway. Arctic Antarctic and Alpine Research 35: 242-247.

TOTLAND $\varnothing$ (1993) Pollination in alpine Norway: flowering phenology, insect visitors, and visitation rates in two plant communities. Canadian Journal of Botany 78: 1072-1079.

UTELLI AB \& BA ROY (2000) Pollinator abundance and behaviour on Aconitum lycoctonum (Ranunculaceae): an analysis of the quantity and quality components of pollination. Oikos 89: 461470.

WALTHER CR (2003) Plants in warmer world Perspectives in Plant Ecology, Evolution and Systematics 6: 169-185.

WARREN SD, KT HARPER \& GM BOOTH (1988) Elevational distribution of insect pollinators. American Midland Naturalist 120: 325-330.

WASER N \& M PRICE (1983) Optimal and actual outcrossing in plants, and nature of plant-pollinator interaction. En: Jones C \& R Little (eds) Handbook of experimental pollination biology: 341-359. Van Nostrand-Reinhold Publishers, New York, New York, USA.

WATKINS L \& DA LEVIN (1990) Outcrossing rates as related to plant density in Phlox drummondii. Heredity 65: 81-89.

WATT VW (1968) Adaptive significance of insect pigment polymorphism in Colias butterflies. I. Variation of melanin pigment in relation to thermoregulation. Evolution 22: 437-458.

WILLMER PG (1983) Thermal constraints on activity patterns in nectar feedings insets. Ecological Entomology 8: 455-469. 